\title{
PROGRAMMIERUNG EINES NAVIGATIONSSYSTEMS FÜR DIE KERNSPINTOMOGRAPHISCHE MAMMADIAGNOSTIK
}

\author{
K. Kühnlenz ${ }^{1}$, J. Oellinger ${ }^{1}$, O. Wendt ${ }^{2}$, U. Boenick ${ }^{2}$ \\ ${ }^{1}$ Universitätsklinikum Charité, Campus Virchow-Klinikum, Strahlen- und Poliklinik \\ ${ }^{2}$ Institut f. Mikro- und Medizintechnik, Technische Universität Berlin
}

tartehbb@sp.zrz.tu-berlin.de

\section{EINLEITUNG}

Die erfolgreiche Therapie karzinomatös erkrankten Gewebes ist in starkem Maße abhängig von einer frühzeitigen Diagnose. Im Bereich der kernspintomographischen Mammadiagnostik stehen hierfür zur Zeit verschiedene diagnostische Methoden, wie z.B. die Stanzbiopsie zur Verfügung. Aufgrund der extremen Magnetfeldumgebung und der engen Platzverhältnisse in Kernspintomographen (KST) müssen im allgemeinen mehrmalige Patientenumlagerungen ausgeführt werden, wobei die Biopsie selbst nur außerhalb des Untersuchungsbereiches und weitgehend manuell durchgeführt wird. Eine exakte Navigation der Biopsienadel in den Zielbereich ist hierbei schwierig und mit Ungenauigkeiten behaftet, so daß karzinomsuspektes Gewebe zum großen Teil nur approximativ biopsiert werden kann. In einem Forschungsprojekt wurde eine Verbesserung dieser Vorgehensweise angestrebt. Eine spezielle Apparatur, welche die automatische Navigation eines medizinschen Instrumentes ermöglicht, wird gemeinsam mit dem Patienten in den Untersuchungsraum (Isozentrum) eines KST positioniert und ermöglicht eine zielgenaue Gewebeentnahme ohne Patientenumlagerungen.

Der Inhalt dieses Berichtes befaßt sich mit der Entwicklung von Softwarestrukturen für dieses System.

\section{MATERIAL UND METHODEN}

Die o.g. Einrichtung läßt sich direkt innerhalb des Untersuchungsbereiches eines KS-Tomographen betreiben und bietet auch gleichzeitig die Aufnahme eines Patienten. Die Zielkoordinatenerfassung und die Stabilisierung der Brust wird mit Hilfe eines spezielles Plattensystems ermöglicht. Für die exakte Positionierung des medizinischen Instrumentes steht ein automatisch gesteuertes Navigationssystem mit laser- und fiberoptischen Sensoren zur Verfügung, welches insgesamt fünf Freiheitsgrade besitzt. Die Signalübertragung von und zu speziellen Meßkarten erfolgt auf Basis von Lichtwellenleitern. Es besteht eine Netzwerkverbindung zwischen dem KS-Tomographen und dem Steuerungssystem. Angewandt wurde z.T. die graphische Programmierumgebung von LABVIEWTM, beispielhaft in Abb.1 gezeigt und die C-orientierte Programmiersprache LABWINDOWS $^{\mathrm{TM}}$ von National Instruments.

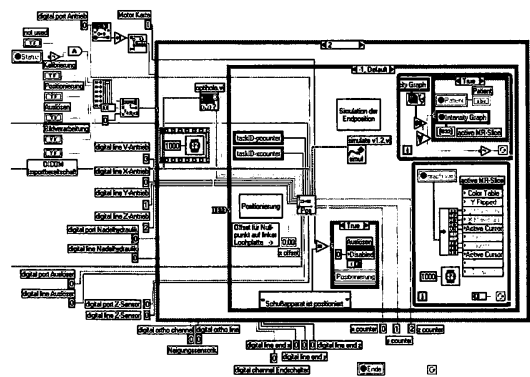

Abb.1: Beispiel für die graphische Entwicklungsumgebung LABVIEW ${ }^{\mathrm{TM}}$ (National Instruments)

Die Abb.2 zeigt die hardwaretechnische Verknüpfung und Anforderungen zwischen dem Kernspintomographen und einem Steuerungsrechner zum Betreiben des automatisierten Navigationssystems.

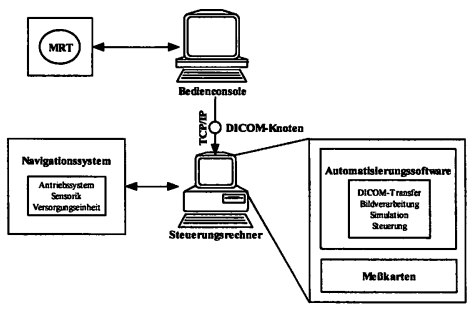

Abb. 2: Allgemeine Struktur des Gesamtsystems

Anzustreben war eine benutzerfreundliche Oberfläche, welche dem Arzt durch suggestive Benutzerführung eine sichere Bedienung und Kontrolle des Systems ermöglichen sollte. Dies wurde durch eine graphische Menüsteuerung und Minimierung des Bedienaufwandes erreicht, so daß die Software einfach anzuwenden ist und zudem schnell erlernt werden kann. Der Programmablauf besitzt eine strenge Hierarchie und ermöglicht eine schrittweise Führung durch die einzelnen Pro- 
grammabschnitte. Auf diese Weise konnte ein Höchst$\mathrm{ma} ß$ an Sicherheit und Reproduzierbarkeit erreicht werden.

\section{FUNKTIONEN DER SOFTWARE}

Nach der Durchführung eines KST-Scans werden ausgewählte Schnittbilder im DICOM- Format über das Klinikumsnetzwerk mittels TCP/IP zum Biopsierechner übertragen. Es folgt eine Decodierung und Konvertierung der Pixelwerte in korrekt wahmehmbare Grauwerte. Dazu werden die im DICOM-Header gespeicherten demographischen Patientendaten, sowie Informationen über die physikalische Geometrie der Schnittbilder ausgelesen, welche für die Berechnung der Zielkoordinaten des Punktionsortes benötigt werden. [1] [2].

Um von den Bildkoordinaten auf physikalische, vom Navigationssystem erreichbare Koordinaten schließen zu können, ist ein gemeinsamer Bezug nötig. Hierzu dient das dem Punktionsort nächstgelegene Plattenelement, in welches Markierungsobjekte KST-aktiver Substanz integriert wurden, die dem Anwender die Plazierung eines Lokalizer im entsprechenden Schnittbild ermöglichen, wie in Abb.3 gezeigt.

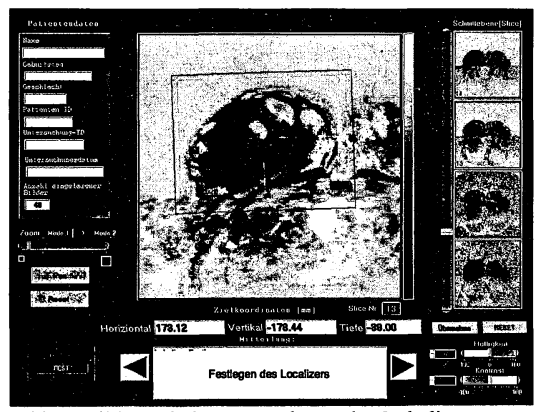

Abb.3: Bildverarbeitung, Festlegen des Lokalizers

Um karzinomsuspekte Gewebeareale identifizieren zu können, stehen eine Reihe von Nachbearbeitungsfunktionen wie Kontrast, Helligkeit und Farbbalance sowie Zoomfunktionen zur Verfügung. Nach erfolgter Identifikation wird der Zielort für die Gewebeentnahme graphisch mit Hilfe eines Fadenkreuzes markiert. Die zur Errechnung der physikalischen Zielkoordinaten erforderlichen Parameter werden daraufhin mittels DDE (Dynamik Data Exchange) an die Steuerungssoftware übertragen. Ein Report, welcher demographische $\mathrm{Pa}$ tientendaten, Zielparameter und Geometrie der Pixeldaten sowie eine Bitmapversion des entsprechenden KST-Bildes enthält, wird daraufhin gesichert.

Anhand der Zielparameter wird nun geprüft, ob der Zielort unter Berücksichtigung der geometrischen Ausprägung der Fixationsplatten und der umgebenden Raumgeometrie erreichbar ist. Hierzu wird die voraussichtliche Lage des Instrumentes in der Biopsieposition berechnet und mit den geometrischen Grenzvorgaben unter Berücksichtigung einer Sicherheitsreserve verglichen. Nadelendposition und Stellung des Instrumentes während der Biopsie werden sodann in einer dreidimensionalen Graphik visualisiert; desweiteren erfolgt die Anzeige von Azimut- und Inklinationswinkel der Biopsienadel (Abb.4). Nach erfolgreicher Simulation kann die Positionierung durchgeführt werden.

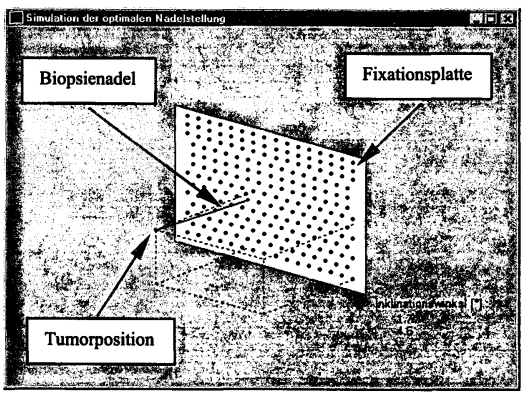

Abb.4: Berechnung und Simulation der Biopsieposition

Vor der exakten Ausrichtung des medizinischen Instrumentes, muß eine Referenzposition erreicht werden, bei der das Instrument orthogonal zur Schnittebene ausgerichtet wird. Die Erfassung dieser Position erfolgt durch Abtastung mittels fiberoptischer Sensoren.

Danach wird das Instrument in eine, dem Zielort optimierte, Position gelenkt. Im nächsten Schritt wird die Einstellung des Azimutes und der Inklination der Nadel vorgenommen. Schließlich kann im Fall einer Biopsie die Eindringtiefe der Nadel justiert werden. Der Arzt kann daraufhin die Punktion sowie den Schuß für die Gewebeentnahme auslösen.

Die Funktion der Software besteht dabei einerseits in der Berechnung der Matrizen für die Koordinatentransformation und weiterhin in der Kommunikation mit den Meßkarten. Mit diesen werden die Signale der Sensorik verarbeitet und die Steuersignale zur Navigation des Instrumentes an die Antriebseinheiten gesendet.

Während des gesamten Ablaufes wird die Position des Instrumentes kontinuierlich im entsprechenden KST-Schnittbild, in welchem die Zielkoordinaten festgelegt wurden, zu Kontrollzwecken visualisiert. Hierzu wird eine Rücktransformation der physikalischen Koordinaten durchgeführt und die 2D-Projektion berechnet.

\section{LITERATURHINWEISE}

[1] The American College of Radiology, Digital Imaging and Communications in Medicine (DICOM) Part 1-4, National Electrical Manufacturers Association, Washington DC, 1993

[2] DICOM - Praktische Aspekte der Bildverarbeitung, Institut für Telematik, Trier, 1998 